\title{
O uso da aromaterapia durante o trabalho de parto: caracterização do conhecimento
}

\section{do enfermeiro}

\author{
The aromatherapy use's during labor: characterization of nurses' knowledge \\ El uso de la aromaterapia durante el trabajo de parto: caracterización del conocimiento del \\ enfermero
}

Recebido: 13/08/2021 | Revisado: 19/08/2021 | Aceito: 20/08/2021 | Publicado: 22/08/2021

\author{
Kamila Martins da Cruz \\ ORCID: https://orcid.org/0000-0001-6741-0205 \\ Universidade Anhanguera, Brasil \\ E-mail: kamila.obstetricia@gmail.com \\ Rosemary Matias \\ ORCID: https://orcid.org/0000-0002-0154-1015 \\ Universidade Anhanguera, Brasil \\ E-mail: rosematiasc@gmail.com \\ Carla Letícia Gediel Rivero-Wendt \\ ORCID: https://orcid.org/0000-0001-6361-6927 \\ Universidade Anhanguera, Brasil \\ E-mail: leticiagediel@gmail.com
}

\begin{abstract}
Resumo
O parto é o momento singular na vida da gestante e a enfermagem com seu modelo assistencial e holístico, consegue colocar a mulher como protagonista do processo de parto, utilizando ferramentas como a aromaterapia. Este estudo caracterizou o conhecimento sobre a aromaterapia e do uso dos óleos essenciais (OEs) durante o trabalho de parto (TP) por meio de um questionário aplicado as enfermeiras das Maternidades Cândido Mariano e do Hospital Universitário Maria Aparecida Pedrossian (HUMAP). A partir dos resultados foi possível verificar que $100 \%$ dos profissionais conhecem a aromaterapia. Entretanto, o percentual $69,2 \%$ possuem formação técnica e conhecimento sobre os meios de diluição e aplicação dos OEs, os profissionais não fazem preferência por marca de produto, $100 \%$ utilizam o óleo essencial (OE) de lavanda e 61,5\% o OE de canela nas parturientes. Assim, a aromaterapia já é uma prática regular entre os enfermeiros das instituições, porém se faz necessário a capacitação adequada, para que apliquem a prática com segurança e respaldo científico.
\end{abstract}

Palavras-chave: Práticas integrativas e complementares; Medicina tradicional; Óleos essenciais; Humanização do parto.

\begin{abstract}
Childbirth is a unique moment in the women pregnant life and the nursing, with its care and holistic model, manages to place the woman as the protagonist of the birth process, using tools such as aromatherapy. This study characterized the knowledge about aromatherapy and the use of essential oils (EOs) during labor (L) through a questionnaire applied to nurses at the Cândido Mariano Maternity and the Maria Aparecida Pedrossian University Hospital (MAPUH) maternity. From the results, it was possible to verify that $100 \%$ of professionals know aromatherapy. However, the percentage of $69.2 \%$ have technical training and the knowledge about the dilution way and application of EOs, the professionals do not prefer product brand, $100 \%$ use lavender essential oil (EO) and $61.5 \%$ use cinnamon EO in parturient. Thus, aromatherapy is already a regular practice among nurses in institutions, but adequate training is necessary, so that they can apply the practice safely and with scientific support.
\end{abstract}

Keywords: Integrative and complementary practices; Traditional medicine; Essential oils; Humanization of childbirth.

\section{Resumen}

El parto es un momento único en la vida de la gestante y la lactancia, con su cuidado y modelo holístico, logra colocar a la mujer como protagonista del proceso del parto, utilizando herramientas como la aromaterapia. Este estudio caracterizó el conocimiento sobre aromaterapia y el uso de aceites esenciales (AE) durante el parto (P) a través de un cuestionario aplicado a enfermeras de las Maternidades Cândido Mariano y del Hospital Universitario María Aparecida Pedrossian (HUMAP). A partir de los resultados se pudo comprobar que el 100\% de los profesionales conocen la aromaterapia. Sin embargo, el porcentaje $69,2 \%$ tiene formación técnica y conocimientos sobre los medios de dilución y aplicación de AE, los profesionales no prefieren la marca del producto, el 100\% utiliza aceite esencial de 
lavanda (AE) y el 61,5\% utiliza el OE Canela en parturientas. Así, la aromaterapia ya es una práctica habitual entre los enfermeros en las instituciones, pero es necesaria una formación adecuada, para que puedan aplicar la práctica de forma segura y con respaldo científico.

Palabras clave: Prácticas integradoras y complementarias; Medicina tradicional; Aceites esenciales; Humanización del parto.

\section{Introdução}

A parturição é um momento marcante e esperado na vida da mulher, pois significa a chegada do bebê e é essencial que neste momento ela receba apoio e cuidado centrado no binômio (mãe e bebê). A World Health Organization - WHO publicou recomendações para cuidados intraparto, que ressaltam o processo de parto como um evento natural, onde o cuidado se torna primordial, constando 56 recomendações baseadas em evidências, 26 novas recomendações adotadas pelo Grupo de Desenvolvimento de Diretrizes (GDG) nas reuniões de 2017 e 30 recomendações existentes relevantes para cuidados intraparto que foram integradas a partir de diretrizes da WHO publicadas anteriormente (Who, 2018).

Dentre as recomendações estão as técnicas não farmacológicas para o alívio da dor, proporcionado para as mulheres grávidas, durante o parto, técnicas manuais com massagens e aplicação de calor dependendo da preferência da parturiente, visando um parto humanizado (Who, 2018).

O parto humanizado adota uma série de práticas e procedimentos envolvendo o respeito à fisiologia natural do corpo (Brasil, 2017) e um dos profissionais que integram a assistência neste período é o enfermeiro, que tem a formação voltada para o cuidado e dispõe sobre o acompanhamento e execução do parto normal sem distocia, fortalecendo o vínculo com a parturiente através de informações, proporcionando um ambiente tranquilo e seguro, com o foco no protagonismo da mulher, que participa ativamente de todo o processo, ajudando a administrar a dor (Silva, Nascimento, \& Coelho, 2015).

A dor durante o TP é uma particularidade de cada mulher e neste momento, se faz necessário o conhecimento do enfermeiro quanto à diversas práticas, para propor medidas de conforto e apoio emocional, dentre elas, a aplicação da aromaterapia (Ficagna, 2020).

Aromaterapia é uma prática que utiliza as essências das plantas aromáticas através dos OEs, que, quando inalados, ativam receptores das vias respiratórias sistema do olfato pelo bulbo e nervos olfativos, que propiciam uma ligação direta com o Sistema Nervoso Central, levando o estímulo ao Sistema Límbico, responsável pelo controle da memória, emoção, sexualidade, impulsos e reações instintivas, proporcionando modificações físicas e psicológicas. Além disso, também pode ser aplicado na pele, penetrando nos ductos das glândulas sebáceas, folículos pilosos e através das células da pele, sendo levados, então, aos receptores sensitivos (Gnatta, Dornellas, \& Silva, 2011).

Por se tratar de uma intervenção não invasiva, não se deve coibir as mulheres que desejarem utilizar a aromaterapia durante o TP. Os métodos não farmacológicos de alívio da dor devem ser oferecidos à mulher antes da utilização de métodos farmacológicos, assegurados pela Política Nacional de Práticas Integrativas e Complementares (PNPIC) no Sistema Único de Saúde (SUS) e pela inclusão da aromaterapia nas práticas integrativas em 2018 (Brasil, 2006, 2018).

As Práticas Integrativas e Complementares (PICs) vêm se disseminando para compor uma assistência humanizada no trabalho de parto e destaca-se a relevância de se conhecer estas práticas na educação em saúde, como a aromaterapia, que são conhecidas e utilizadas por enfermeiros nas salas de partos. Assim, o objetivo do estudo foi caracterizar o conhecimento sobre a aromaterapia e do uso de óleos essenciais (OEs) durante o trabalho de parto (TP) sob o olhar do profissional de enfermagem, de duas instituições de Campo Grande - MS. 


\section{Metodologia}

Realizou-se um estudo descritivo e transversal com abordagem quanti-qualitativa (Pereira et al., 2018), na maternidade (Maternidades Cândido Mariano) e na maternidade de Hospital (Hospital Universitário Maria Aparecida Pedrossian), localizados no município de Campo Grande, MS.

A coleta de dados ocorreu no período entre dezembro de 2020 a janeiro de 2021. O instrumento da pesquisa (um questionário composto por 18 questões abertas e fechadas) foi aplicado nas enfermeiras atuantes na assistência direta ao parto, com duração de aplicação de no máximo 10 minutos.

O intuito foi caracterizar o conhecimento dos enfermeiros sobre o uso dos OEs, da aromaterapia, aquisição do conhecimento especializado e da importância da profissão para divulgação e popularização da terapia. Foram convidados todos os enfermeiros, 16 profissionais, que trabalhavam na sala de trabalho de parto das respectivas instituições. Não responderam à pesquisa os profissionais que estavam de férias ou licença, desta forma, a amostra foi constituída por 13 enfermeiras.

Os resultados foram tabulados em planilha eletrônica Microssoft Office Excel ${ }^{\circledR}$, apresentados em forma de gráficos, analisados pela estatística descritiva, com frequência absoluta, porcentagem e discutidos a partir da literatura pertinente.

O projeto de pesquisa foi aprovado pelo Comitê de Ética em Pesquisa de Seres Humanos da Universidade Anhanguera-Uniderp, sob o CAAE $\mathrm{n}^{\circ}$. 40687320.8.0000.5161. Todos os participantes da pesquisa assinaram o termo de consentimento livre e esclarecido - TCLE.

\section{Resultados e Discussão}

Descrição quantitativa sobre os enfermeiros e seus conhecimentos sobre as práticas integrativas e complementares, aromaterapia e óleos essenciais

Por meio da pesquisa, foi verificado que no HUMAP o quadro de enfermeiros que atuam na sala de parto é composto por $12(100 \%)$ e na maternidade Cândido Mariano são um total de 4 (100\%) profissionais.

Em relação a maternidade Cândido Mariano foi aplicado para 100\% das enfermeiras e no HUMAP, o questionário foi aplicado para 9 enfermeiras, representando $75 \%$ do efetivo total.

Da amostra coletada observou-se que em ambas as instituições 100\% dos indivíduos foram do sexo feminino e 53,8\% foram representados na faixa etária entre 30 e 35 anos, estando de acordo com a história do surgimento da profissão, pois a assistência às parturientes era realizada por parteiras, atividade destinada exclusivamente às mulheres (De Sena, Santos, Carvalho, Sá, \& Paixão, 2012).

Na pesquisa, todas as enfermeiras têm conhecimento sobre as práticas integrativas no atendimento à gestante em TP e conhecem a aromaterapia e 76,9\% aplicam seus conhecimentos em suas pacientes. Entretanto, apenas 69,2\% possuem conhecimento técnico sobre a aromaterapia e o uso dos OEs.

O último dado evidencia a necessidade de capacitar os profissionais para aquisição dos saberes técnicos sobre a aplicação das PICs (Cofen, 2015), corroborando com uma assistência que seja livre de danos e para que possam encontrar nas terapias, a possibilidade de trazer benefícios às parturientes (Azevedo et al., 2019) e para o profissional da enfermagem, que segundo o Coren/SC, os profissionais de Enfermagem tem amparo legal para realizar as PICS, de acordo com o Código de Ética dos profissionais de Enfermagem. Para o Conselho Federal de Enfermagem (COFEN), na Resolução COFEN $n^{\circ}$ 564/2017 (Cofen, 2017), o profissional de Enfermagem por atuar na promoção, restauração da saúde, prevenção de agravos e doenças, quando possui competência profissional e especializada no assunto, mediante comprovação de titularidade, contempla o uso da aromaterapia (Lara, Matias, Oliveira, Facco, \& Vieira, 2020). 
Quando questionadas sobre os meios utilizados para aquisição dos saberes das práticas descritas, 62\% das enfermeiras adquiriram seus conhecimentos por cursos online sobre as PICs e 39\% responderam que os conhecimentos vieram de curso de pós-graduação lato sensu em obstetrícia (Figura 1).

Figura 1. Representação dos meios de aquisição de conhecimento sobre as Práticas Integrativas e Complementares das 13 Enfermeiras participantes da pesquisa da Maternidade Cândido Mariano ( $n=4)$ e Hospital Universitário (n=9), Campo Grande - MS.

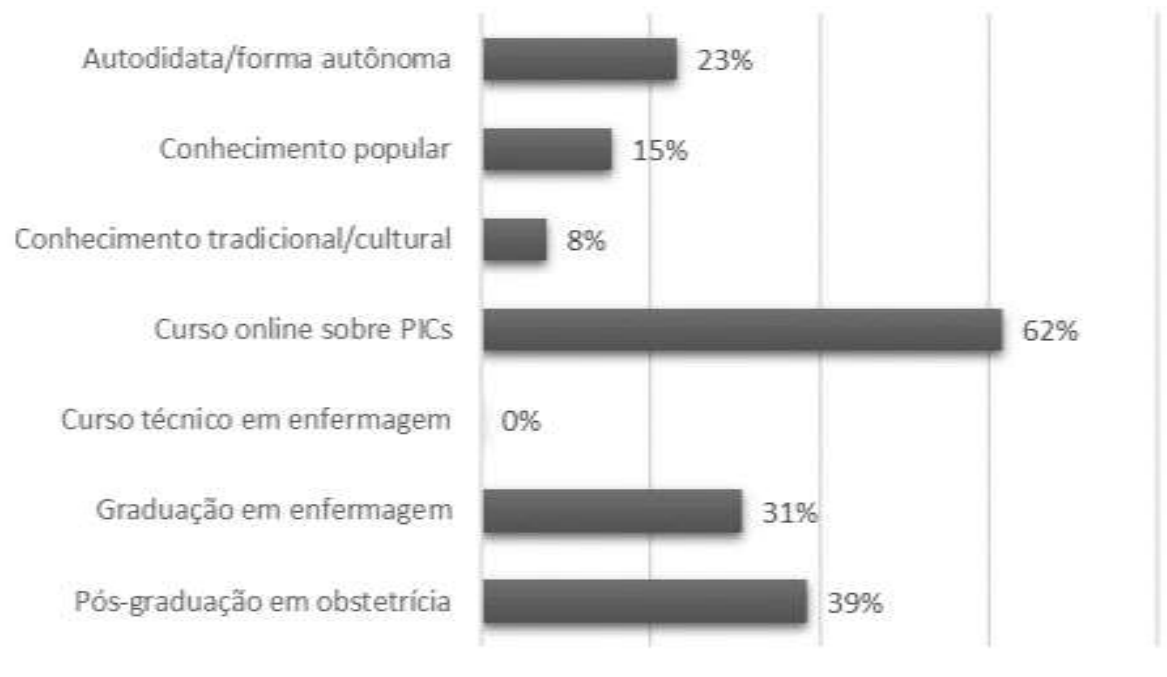

Fonte: Autores.

Os dados descritos na Figura 1, demonstram que os enfermeiros possuem conhecimento quanto à aromaterapia e que não foram repassados de forma exclusiva em suas formações. Os impasses relativos à inclusão das PICs nas propostas pedagógicas das instituições de ensino repercutem na implantação efetiva das PICs no SUS, devido ao déficit na formação de profissionais e à limitada produção de estudos científicos referentes à efetividade das mesmas. Assim, dificulta a aplicação do novo modelo de atenção à saúde da mulher e a inserção de profissionais terapeutas legitimados no mercado de trabalho (Barbosa et al., 2006; Fontanella, Speck, Piovezan, \& Kulkamp, 2007).

O uso da PICs quando bem exercidas pelo enfermeiro podem proporcionar benefícios como a redução da dor e ansiedade, colocando a mulher como protagonista do parto, empoderando - a, desenvolvendo autonomia e autocuidado consciente de tudo o que acontece com seu corpo nas fases do parto (Borges, Madeira, \& Azevedo, 2011). Na pesquisa sobre as PICs, $100 \%$ das enfermeiras apontaram conhecer a aromaterapia e musicoterapia, seguido por 39\% que conheciam a acupuntura, fitoterapia, homeopatia e $31 \%$ conhecem sobre o uso das plantas medicinais (Figura 2). 
Figura 2. Representação do conhecimento sobre as práticas integrativas e complementares utilizadas na gestante e no trabalho de parto, resposta de 13 Enfermeiras participantes da pesquisa da Maternidade Cândido Mariano (n= 4) e Hospital Universitário (n=9), Campo Grande - MS.

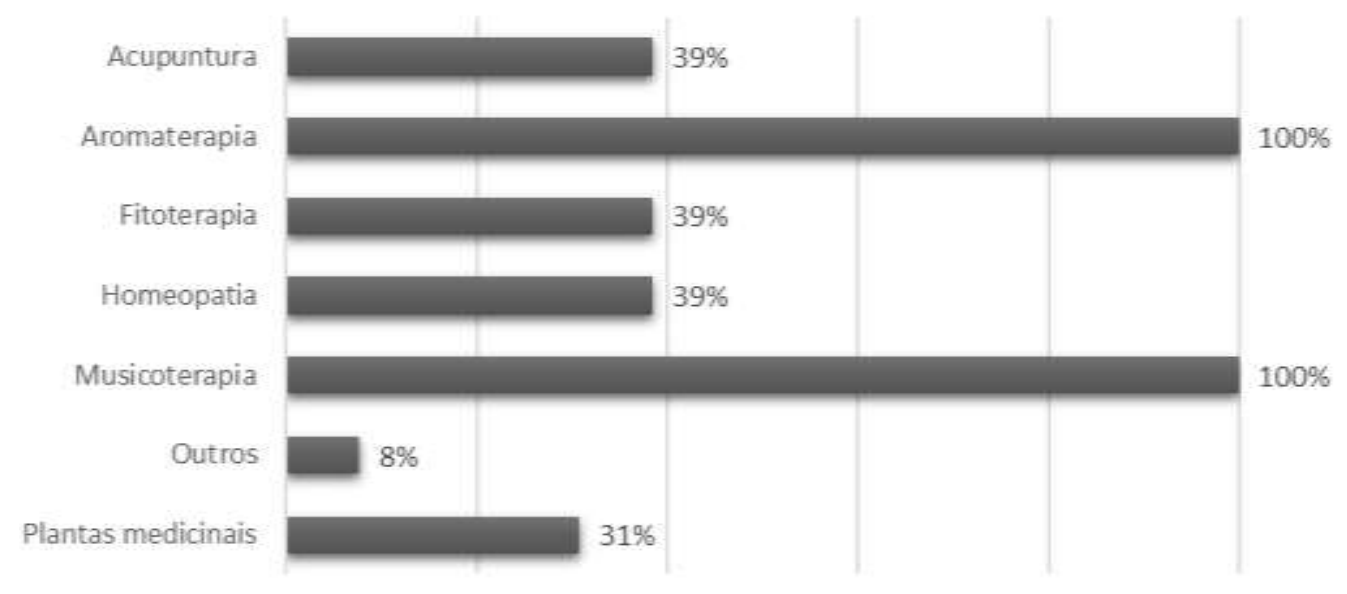

Fonte: Autores.

A aromaterapia tem se mostrado uma valiosa ferramenta para a prática do profissional enfermeiro obstetra em sua atuação com métodos não farmacológicos, para que se obtenha o alívio da dor durante do TP (Souza et al., 2019).

A aplicação da aromaterapia vai além do tratamento, ela se faz valer dos poderes dos OEs para predispor não só à cura, mas também à prevenção e equilíbrio psicossomático do organismo. Para o sucesso e eficácia do procedimento, a aromaterapia depende da ação dos OEs por meio do contato com a pele, a corrente sanguínea e sistema olfativo (Andrei \& Comune, 2005).

O modo de aplicação dos OEs ativa o sistema límbico e alteram as catecolaminas (Nursahidah, Novelia, \& Suciawati, 2020). De acordo com o relatado por $92,3 \%$ das enfermeiras, que evidenciam a diminuição do estresse e ansiedade, assim como atenuação das dores inerentes ao TP quando utilizado a aromaterapia em suas clientes. Dos benefícios especificados, $100 \%$ observam relaxamento, 69,2\% diminuição da ansiedade e 46,2\% melhor controle da dor e aumento da confiança da parturiente.

O efeito benéfico e a eficácia da aromaterapia estão relacionados às fases do TP em que é aplicada. No estudo, das enfermeiras que utilizam os OEs, todas aplicam durante a fase ativa, 30,8\% durante a fase latente e $15,4 \%$ durante o período expulsivo. Os dados coincidem com o uso do óleo de rosa damasco na diminuição da severidade da dor e da ansiedade das parturientes durante a fase ativa do trabalho de parto (Hamdamian et al., 2018; Kheirkhah, Setayesh Vali Pour, Nisani, \& Haghani, 2014)

Em relação à forma de utilização, diluição e aquisição do produto, 69,2\% têm conhecimento e todas informaram ser importante a qualidade do produto em relação à pureza dos OEs. Dentre as marcas informadas na pesquisa, a mais utilizada é a Bioessência (39\%), seguida pela Do Terra e Terra flor (23\%), porém 46\% responderam outras, justificando como qualquer marca ou várias delas (Figura 3). 
Figura 3. Registro das marcas de fabricante de óleos essenciais utilizados durante o trabalho de parto, resposta das 13 Enfermeiras participantes da pesquisa da Maternidade Cândido Mariano (n=4) e Hospital Universitário (n= 9), Campo Grande - MS.



Fonte: Autores.

A pureza do óleo essencial é uma peça-chave para que a aplicação da aromaterapia seja segura (Naha, 2017), entretanto não há garantias sobre a avaliação de medição da pureza pelo fabricante (Manion \& Widder, 2017). O uso dos óleos essenciais sem a verificação do percentual de pureza e real aplicabilidade durante a gestação e parto, pode implicar em complicações para a gestante ou parturiente, pois alguns óleos essenciais atravessam a barreira placentária (Ferraz \& Avellar, 2020). O óleo essencial de lavanda, por exemplo, é capaz de diminuir a dor durante o parto, além do estresse e ansiedade da parturiente, favorecendo a concentração e tranquilidade (Souza et al., 2019), porém, quando utilizado com percentuais acima de $15 \%$ dos metabólitos de linalol e acetato de linalila, são contraindicados no TP e o seu uso deve ser feito com cautela na gestação e durante o parto (Ferraz \& Avellar, 2020). No questionário, 100\% das enfermeiras responderam que utilizam o óleo de lavanda e $62 \%$ o de canela (Figura 4$)$. 
Figura 4. Tipos de óleos essenciais utilizados durante o trabalho de parto, resposta das 13 Enfermeiras participantes da pesquisa da Maternidade Cândido Mariano (n=4) e Hospital Universitário (n=9), Campo Grande - MS.

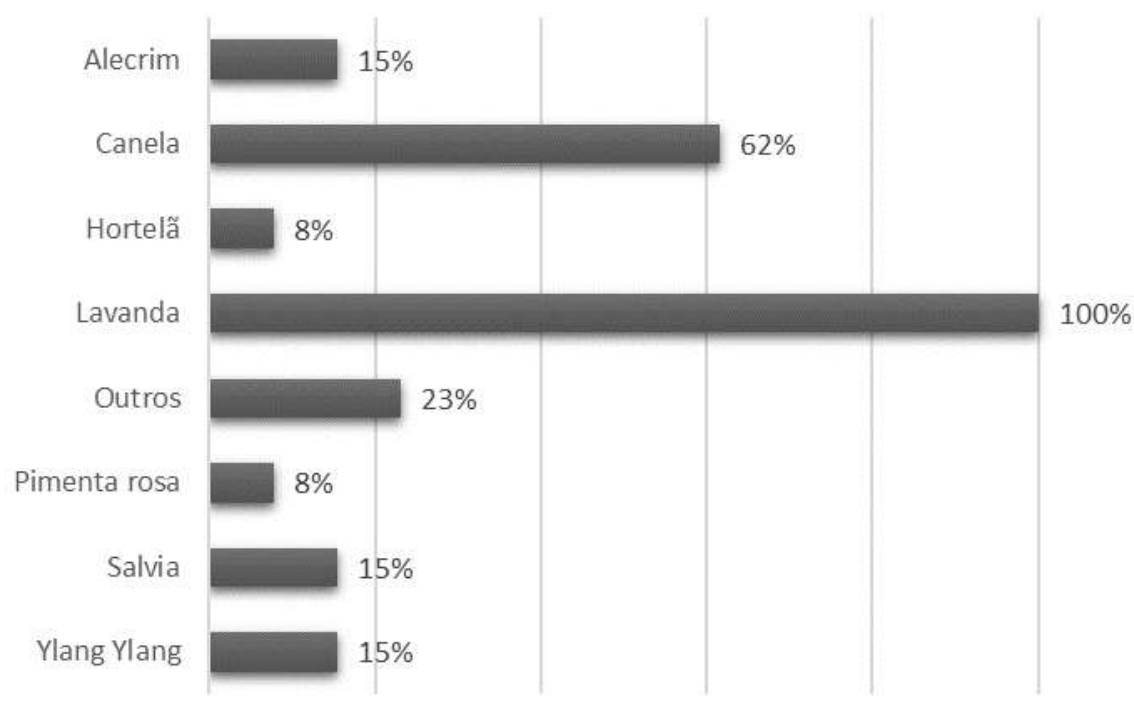

Fonte: Autores.

Os OEs têm eficácia devido às suas propriedades analgésicas, sedativas, anti-inflamatórias, antioxidantes e antimicrobianas que estão relacionadas aos compostos químicos, agindo no hipotálamo e ativando o sistema nervoso parassimpático, estimulando a ação dos hormônios femininos durante o trabalho de parto (Kartilah \& Februanti, 2020; Sumarni, Astuti, \& Karmilah, 2019; Wells, Truong, Adal, Sarker, \& Mahmoud, 2018).

O OE canela (Cinnamomum verum) é um exemplo de que é preciso ter cautela no uso da aromaterapia. Ele foi apontado pelas enfermeiras como sendo o segundo mais utilizado no TP sendo que, pela literatura científica, sua utilização deverá ser cuidadosa, não podendo utilizar o OE puro por ser cáustico, causando irritação cutânea. No TP têm finalidade de aumentar as contrações, por aumentar a circulação sanguínea e ocasionar vasodilatação (Cardoso et al., 2010; Ferraz \& Avellar, 2020; Rodrigues, Ferreira, \& Andrade, 2018).

As enfermeiras ressaltaram sobre a importância da utilização dos OEs através da aromaterapia no trabalho de parto e destacaram o papel do enfermeiro na aplicação e divulgação sobre o conhecimento da prática entre os profissionais da enfermagem e gestantes.

\section{Conhecimento acerca da importância do enfermeiro na disseminação e uso da aromaterapia durante o trabalho de parto}

As enfermeiras ressaltaram sobre quão importante é o seu papel atuando na aplicação dos métodos em pacientes atendidos na maternidade do HUMAP e na Maternidade Cândido Mariano, destacando o enfermeiro como responsável pelo conforto e segurança da paciente, por transmitir o conhecimento técnico para a equipe, paciente, acompanhante e para as técnicas de enfermagem, sobre os métodos não farmacológicos para o alívio da dor da parturiente.

Respostas sobre a importância do enfermeiro:

[...] tem o papel importante trazendo conforto e segurança a paciente (Enf 1).

[...] de grande importância para implementação dessa prática na assistência, para difundir os benefícios desse método entre os profissionais e para benefício do cliente (Enf 2). 
[...] O Enfermeiro é a peça fundamental para que a aromaterapia seja difundida durante o trabalho de parto, sem ele está técnica não seria aplicada (Enf 3).

[...] Extrema importância a replicação do conhecimento e da informação tendo em vista os benéficos trazidos na assistência (Enf4).

[...] no pré-natal orientar que existe e como pode auxiliar no processo de trabalho de parto, no intraparto esclarecer qual seria indicado e por quêl como ele pode ajudar (Enf 8).

[...] Pela proximidade desta categoria com as gestantes e parturientes, tanto no pré-natal, trabalho de parto e parto fica mais fácil de orientar os benefícios e auxiliar na utilização (Enf 11).

[...] Papel importantíssimo, pois, normalmente é esse profissional que apresenta e aplica esses métodos nas pacientes atendidas no HUMAP (Enf 12).

O enfermeiro destaca-se como o profissional que escuta atentamente as necessidades da parturiente pelo domínio do diálogo-interação obtido pela educação em saúde. O profissional da enfermagem busca esclarecer dúvidas e procedimentos técnicos necessários ao acompanhamento e evolução clínica da mulher parturiente (Almeida et al., 2018; Trindade et al., 2020).

A experiencia do TP se torna mais difícil quando a mulher está sozinha ou sem acompanhante da família, destacando sentimentos como a ansiedade, angústia e medo. Sem a assistência à parturiente em todas suas demandas, incluindo a emocional, é possível que a mulher tenha sensações de pânico, falta de controle e até alterações nas contrações uterinas. O parto ocorre de maneira mais natural quando a mulher se sente segura e relaxada estando próxima à pessoa escolhida para ficar com ela no momento do parto (Melo et al., 2011).

O sentimento de medo da parturiente no momento de escolher o tipo de parto é um dos motivos que incentivaram o Ministério da Saúde a criar as Diretrizes Nacionais de Assistência ao Parto Normal (Brasil, 2017). As diretrizes qualificam a assistência prestada à gestante contribuindo para o cuidado humanizado, diminuindo traumas e riscos (Velho et al., 2019).

$\mathrm{O}$ uso de medidas não farmacológicas é abordado nas diretrizes para o alívio da dor durante o TP, dentre elas estão a imersão em água, massagens, musicoterapia e aromaterapia (Brasil, 2017).

A aromaterapia, segundo a pesquisa de opinião aplicada, deve ser implementada na prática de assistência para difundir os benefícios entre os profissionais e usuários. Os benefícios são observados pela equipe de saúde, que devem orientar as mães no pré-natal sobre a existência da terapia e durante o TP. A mulher gestante se empodera durante o TP sob a orientação, esclarecimento e apoio do enfermeiro.

Dentre os benefícios apontados, as enfermeiras ressaltaram que a técnica:

[...] tem o papel importante trazendo conforto e segurança a paciente (Enf 5).

[...] levar conhecimento técnico para equipe, paciente e acompanhante, a resposta das técnicas não farmacológicas para alívio da dor para melhor atendimento frente ao processo de trabalho de parto (Enf 6).

[...] mostra através da oferta do aroma e utilização dos benefícios, sentidos pelas mães e observados pela equipe de saúde (Enf 7).

[...] A melhoria no trabalho de parto (Enf 9).

[...] Orientação, esclarecimento e apoio no empoderamento da gestante o trabalho de parto (Enf 10). 
O uso de práticas terapêuticas não farmacológicas, como a aromaterapia, está se consolidando em Centros de Parto Normal, com a humanização das ações de enfermeiras, médicos e outros profissionais envolvidos no atendimento às mulheres, assim o profissional de enfermagem reconhece e valoriza a utilização das tecnologias no contexto do parto como prática do seu cuidado junto as parturientes (Duarte et al., 2019).

As respostas mencionadas no estudo identificaram que o profissional da enfermagem atuante há tempos na assistência aos partos, precisa se atualizar nas práticas através do conhecimento específico, ressaltando que o papel do enfermeiro é fundamental devido à proximidade das gestantes e parturientes, tanto no pré-natal, TP e parto, sendo mais fácil orientar sobre os benefícios e auxiliar na utilização dos OEs e aromaterapia.

Sobre o processo de capacitação e atualização do enfermeiro para aplicar as técnicas e a aromaterapia foram respondidas:

\section{[...] Os enfermeiros que atuam a muito tempo na assistência precisam estar em constante atualização de suas práticas através do conhecimento (Enf 4).}

A World Health Organization e o Ministério da Saúde no Brasil vêm implantando ações para a humanização da saúde, como apoio ao fortalecimento da formação e atuação através de cursos de aprimoramento de enfermeiras em todo o país (Brasil, 2017; Who, 2018). Neste contexto, a profissão de assistência à saúde à parturiente se consolida e se respalda para melhor beneficiar a cliente.

\section{Conclusão}

A prática da aromaterapia é conhecida pelos enfermeiros das instituições descritas e que atuam nas salas de parto. Entretanto, o profissional necessita de capacitação sobre o uso dos óleos essenciais para obter resultados benéficos à parturiente.

As enfermeiras acreditam que a qualidade do produto é essencial para segurança e boa prática, mas não especificam uma marca, o que nos leva a concluir que o uso dos óleos essenciais durante o trabalho de parto deve ser popularizado dentro do respaldo científico.

Os profissionais conhecem diferentes óleos essenciais, como o de lavanda que possui propriedades analgésicas e ansiolíticas já estudas, entretanto, o óleo essencial de canela, o segundo mais utilizado pelas enfermeiras, pode provocar reações indesejadas na parturiente de acordo com a aplicação.

Sendo assim, a ampla divulgação do tema e sustentação científica sobre os efeitos dos óleos essenciais pode favorecer a implantação permanente do uso da técnica nos centros obstétricos do estudo, e na assistência de todos os profissionais que atuam na área, levando os benefícios e tornando a relação do profissional com a parturiente mais consolidada, proporcionando segurança e controle emocional durante o TP e parto.

\section{Agradecimentos}

Às instituições que autorizaram que a pesquisa fosse realizada com sua equipe, Hospital Universitário Maria Aparecida Pedrossiam e Maternidade Cândido Mariano e a Universidade Anhanguera-Uniderp, pela bolsa de estudo.

\section{Referências}

Almeida, J. R. de, Vianini, M. C. dos S., Silva, D. M., Meneghin, R. A., Souza, G. de, \& Resende, M. A. (2018). O enfermeiro frente às práticas integrativas e complementares em saúde na estratégia de saúde da família. Revista Eletrônica Acervo Saúde, (18), e77. https://doi.org/10.25248/reas.e77.2019 
Andrei, P., \& Comune, A. P. Del. (2005). Aromaterapia e suas aplicações Aromatherapy and its applications. Cadernos, 11, 57-68.

Azevedo, C., Moura, C. de C., Corrêa, H. P., Mata, L. R. F. da, Chaves, É. de C. L., \& Chianca, T. C. M. (2019). Complementary and integrative therapies in the scope of nursing: legal aspects and academic-assistance panorama. Escola Anna Nery, 23(2), 1-9. https://doi.org/10.1590/2177-9465-ean-2018-0389

Barbosa, M. A., Martins Fonseca, A. P., Bachion, M. M., Souza, J. T. de, Faria, R. M., Malagoni de A C Oliveira, L., \& Maria Silva Andraus, L. (2006). Terapias alternativas de saúde $\mathrm{x}$ alopatia: Tendências entre acadêmicos de medicina. Revista Eletrônica de Enfermagem, 3(2). https://doi.org/10.5216/ree.v3i2.718

Borges, M. R., Madeira, L. M., \& Azevedo, V. M. G. de O. (2011). As práticas integrativas e complementares na atenção à saúde da mulher: uma estratégia de humanização da assistência no Hospital Sofia Feldman. Revista Mineira de Enfermagem, 15(1), $105-113$.

Brasil. (2006). Política Nacional de Práticas Integrativas e Complementares no SUS: uma ação de inclusão. In Ciência \& Saúde Coletiva (Vol. 11).

Brasil. (2017). Diretrizes Nacionais de Assistência ao Parto Normal. Ministério da Saúde.

Brasil. Portaria nº 702, de21 de março de 2018. , Ministério da Saúde § (2018).

Cardoso, M. G., Gavilanes, M. L., Marques, M. C. S., Shan, A. Y. K. V., Santos, B. R., Oliveira, A. C. B., \& Pinto, A. P. S. (2010). Óleos Essenciais. In L. A. . P. P. R. Simões, C. M. O., Schenkel, E. P., Gosmann, G., Mello, J. C. P., Mentz (Ed.), Farmacognosia: da planta ao medicamento. (6th ed.), $467-495$. Editora da UFRGS.

Cofen. Resolução Cofen n $n^{o}$ 500/2015, Pub. L. No. 500 (2015). Conselho Federal de Enfermagem.

Cofen. Resolução COFEN No 564/2017. (2017).

De Sena, C. D., Santos, T. C. S., Carvalho, C. M. F., Sá, A. C. de M., \& Paixão, G. P. do N. (2012). Avanços e retrocessos da enfermagem obstétrica no Brasil. Revista de Enfermagem Da UFSM, 2(3), 523-529. https://doi.org/10.5902/217976923365

Duarte, M. R., Alves, V. H., Rodrigues, D. P., de Souza, K. V., Pereira, A. V., \& Pimentel, M. M. (2019). Care technologies in obstetric nursing: Contribution for the delivery and birth. Cogitare Enfermagem, 24. https://doi.org/10.5380/ce.v24i0.54164

Ferraz, A., \& Avellar, M. (2020). Aromaterapia na gestação. Viver de Aromas.

Ficagna, F. T. (2020). Profissionais Da Saúde Em Hospitais Públicos Para O Alívio Da Dor No Trabalho De Parto : Non-Pharmacological Methods Used By Health Professionals in Public. 9.

Fontanella, F., Speck, F. P., Piovezan, A. P., \& Kulkamp, I. C. (2007). Conhecimento, acesso e aceitação das práticas integrativas e complementares em saúde por uma comunidade usuária do Sistema Único de Saúde na cidade de Tubarão/SC TT - knowledge, access and acceptance referring to complementary and integrative practices i. ACM Arq. Catarin. Med, 36, 69-74.

Gnatta, J. R., Dornellas, E. V., \& Silva, M. J. P. da. (2011). O uso da aromaterapia no alívo da ansiedade. Acta Paulista de Enfermagem, 24(2), 257-263. https://doi.org/10.1590/S0103-21002011000200016

Hamdamian, S., Nazarpour, S., Simbar, M., Hajian, S., Mojab, F., \& Talebi, A. (2018). Effects of aromatherapy with Rosa damascena on nulliparous women's pain and anxiety of labor during first stage of labor. Journal of Integrative Medicine, 16(2), 120-125. https://doi.org/10.1016/j.joim.2018.02.005

Kartilah, T., \& Februanti, S. (2020). Effectiveness of progressive muscle relaxation and aromatherapy on fatigue in pregnant mothers. Medisains, 18(1), 14. https://doi.org/10.30595/medisains.v18i1.6421

Kheirkhah, M., Setayesh Vali Pour, N., Nisani, L., \& Haghani, H. (2014). Comparing the effects of aromatherapy with rose oils and warm foot bath on anxiety in the first stage of labor in nulliparous women. Iranian Red Crescent Medical Journal. https://doi.org/10.5812/ircmj.14455

Lara, S. D., Matias, R., Oliveira, A. k. M., Facco, G. G., \& Vieira, S. C. H. (2020). Boletim Técnico: Práticas Integrativas e Complementares - PICS no SUS e a Atividade do Enfermeiro (p. 25). p. 25. Londrina: Unopar.

Manion, C. R., \& Widder, R. M. (2017). Essentials of essential oils. American Journal of Health-System Pharmacy, 74(9), e153-e162. https://doi.org/10.2146/ajhp151043

Melo, J. S. de, Kerber, N. P. da C., Oliveira, A. M. N. de, Busanello, J., Silveira, T. S. da, \& Pohlmann, F. C. (2011). Inserção do acompanhante no cuidado da adolescente em um centro obstétrico do sul do país1. Ciência, Cuidado e Saúde, 10(4), 781-788. https://doi.org/10.4025/cienccuidsaude.v10i4.18323

Naha. (2017). Exploring aromatherapy: safety information. National Association for Holistic website: https://naha.org/explore-aromatherapy/safety

Nursahidah, A. A., Novelia, S., \& Suciawati, A. (2020). The Effect of Lavender Aromatherapy on Labor Pain Among Delivery Women in Bandung 2020. Asian Community Health Nursing Research, 2(1), 13-19.

Pereira, A. S., Shitsuka, D. M., Parreira, F. J., \& Shitsuka, R. (2018). Metodologia da Pesquisa Científica (1a.). Santa Maria, RS: Universidade Federal de Santa Maria.

Rodrigues, E. S., Ferreira, I. P., \& Andrade, M. A. (2018). Protocolo de plantas medicinais e fitoterápicos na assistência obstétrica. Belém.

Silva, A. L. S., Nascimento, E. R. do, \& Coelho, E. de A. C. (2015). Nurses practices to promote dignity, participation and empowerment of women in natural childbirth. Escola Anna Nery - Revista de Enfermagem, 19(3), 424-431. https://doi.org/10.5935/1414-8145.20150056 
Research, Society and Development, v. 10, n. 11, e68101119417, 2021

(CC BY 4.0) | ISSN 2525-3409 | DOI: http://dx.doi.org/10.33448/rsd-v10i11.19417

Souza, F. M. D. L. C., Dos Santos, W. N., Santos, R. S. D. C., Rodrigues, O. B., Santiago, J. D. C. D., \& Da Silva, R. A. R. (2019). Tecnologias Apropriadas Ao Processo Do Trabalho De Parto Humanizado. Enfermagem Em Foco, 10(2). https://doi.org/10.21675/2357-707x.2019.v10.n2.2180

Sumarni, R., Astuti, I., \& Karmilah, N. (2019). the Relations of Aromatherapy With the Intensity of Pain on Labor Women Stage I Active Phase in Pmb Cimahi City. Third International Seminar on Global Health (3rd ISGH), 3(1), 21-26.

Trindade, C. D., Cardoso, L. S., Costa, V. Z., Rosa, L. S., Pozzobon, D. M., Trindade, L. R., \& Ambrós, E. L. (2020). Equipe de Enfermagem: a comunicação na assistência à parturiente. Brazilian Journal of Health Review, 3(1), 551-562. https://doi.org/10.34119/bjhrv3n1-043

Velho, M. B., Brüggemann, O. M., McCourt, C., Gama, S. G. N. da, Knobel, R., Gonçalves, A. de C., \& d'Orsi, E. (2019). Modelos de assistência obstétrica na Região Sul do Brasil e fatores associados. Cadernos de Saude Publica, 35(3), e00093118. https://doi.org/10.1590/0102-311X00093118

Wells, R., Truong, F., Adal, A. M., Sarker, L. S., \& Mahmoud, S. S. (2018). Lavandula essential oils: A current review of applications in medicinal, food, and cosmetic industries of lavender. Natural Product Communications, 13(10), 1403-1417. https://doi.org/10.1177/1934578x1801301038

Who. (2018). WHO recommendations: intrapartum care for a positive childbirth experience. World Organozation Health. 